\title{
THE GAU-WANG-WU CONJECTURE ON PARTIAL ISOMETRIES HOLDS IN THE 5-BY-5 CASE*
}

\author{
ILYA M. SPITKOVSKY ${ }^{\dagger}$, IBRAHIM SULEIMAN ${ }^{\dagger}$, AND ELIAS WEGERT ${ }^{\ddagger}$
}

\begin{abstract}
Gau, Wang and Wu in their LAMA'2016 paper conjectured (and proved for $n \leq 4$ ) that an $n$-by- $n$ partial isometry cannot have a circular numerical range with a non-zero center. We prove that this statement holds for $n=5$.
\end{abstract}

Key words. Numerical range, Partial isometry, Kippenhahn curve, Kippenhahn polynomial.

AMS subject classifications. 15A60, 47A12.

1. Introduction. Let $A$ be a bounded linear operator acting on a Hilbert space $\mathcal{H}$. The numerical range (a.k.a. the field of values, or the Hausdorff set) of $A$ is defined as

$$
W(A):=\{\langle A x, x\rangle: x \in \mathcal{H},\|x\|=1\}
$$

where $\langle.,$.$\rangle stands for the scalar product on \mathcal{H}$, and $\|$.$\| is the respective norm. Clearly, W(A)$ is a subset of $\{z \in \mathbb{C}:|z| \leq\|A\|\}$, closed in the case $\operatorname{dim} \mathcal{H}<\infty$ (but not necessarily in general) and invariant under unitary similarities of $A$. By the celebrated Toeplitz-Hausdorff theorem, $W(A)$ is always convex - see e.g. [2] or [3, Chapter 1] and the references therein for a comprehensive treatment of the subject.

An operator $A$ is a partial isometry if it preserves norms of the vectors from the orthogonal complement of its kernel:

$$
\|A x\|=\|x\| \text { whenever } x \in \operatorname{ker}(A)^{\perp} .
$$

It was conjectured by Gau, Wang, and $\mathrm{Wu}[1]$ that

If $A$ is a partial isometry acting on a finite-dimensional space $\mathcal{H}$ and $W(A)$ is a circular disk, then the latter is necessarily centered at the origin.

In what follows, we will refer to this statement as (GWW). According to [1, Theorem 2.2], (GWW) holds for $\operatorname{dim} \mathcal{H}=: n \leq 4$. To the best of our knowledge, the question remains open for higher values of $n$. Very recently, the case $n=5$ was treated in [6] under some additional restrictions on $A$. The special case where $\operatorname{dim} \operatorname{ker} A=1$ was settled by two of us in [7] for any $n$. In the paper at hand, we show that (GWW) holds unconditionally for $n=5$.

THEOREM 1.1. Let A be a partial isometry acting on a 5-dimensional space and assume that its numerical range $W(A)$ is a circular disk. Then this disk is centered at the origin.

\footnotetext{
${ }^{*}$ Received by the editors on August 21, 2021. Accepted for publication on January 18, 2022. Handling Editor: Tin-Yau Tam. Corresponding Author: Ilya M. Spitkovsky

$\dagger$ Division of Science and Mathematics, New York University Abu Dhabi (NYUAD), Saadiyat Island, P.O. Box 129188 Abu Dhabi, United Arab Emirates (ims2@nyu.edu, is1647@nyu.edu). The results are partially based on the Capstone project of the second named author under the supervision of the first named author. The latter was also supported in part by Faculty Research funding from the Division of Science and Mathematics, New York University Abu Dhabi.

${ }^{\ddagger}$ Institute of Applied Analysis, TU Bergakademie Freiberg, 09596 Freiberg, Germany (wegert@math.tu-freiberg.de).
} 
2. General remarks. Denoting by $\mathcal{R}(A)$ the range of an operator $A$, observe that the closure $\mathcal{L}$ of $\mathcal{R}(A)+\mathcal{R}\left(A^{*}\right)$ is a reducing subspace of $A$. Let $A_{0}$ stand for the restriction of $A$ onto $\mathcal{L}$; the restriction of $A$ onto $\mathcal{L}^{\perp}=\operatorname{ker}(A) \cap \operatorname{ker}\left(A^{*}\right)$ is the zero operator. Note that $\mathcal{R}(A)+\mathcal{R}\left(A^{*}\right)$ is automatically closed if $A$ has finite rank - the only case we are interested in. If $A$ is a partial isometry, then $A_{0}$ is a partial isometry as well. If, in addition, $W(A)$ is a circular disk, then so is $W\left(A_{0}\right)$. Since $\operatorname{dim} \mathcal{L} \leq 2 \operatorname{rank} A$, [1, Theorem 2.2] immediately implies the following

Proposition 2.1. (GWW) holds for partial isometries of rank one and two, independently of the dimension of $\mathcal{H}$.

Note also that $(\mathrm{GWW})$ is tautologically correct when $\operatorname{dim} \mathcal{L}=\operatorname{rank} A$ is finite. Indeed, in this case, $A_{0}$ is unitary, and so $W\left(A_{0}\right)$ and $W(A)$ are polygons, never circular disks. When $n=5$, we therefore only need to consider two remaining values of $\operatorname{rank} A$, namely 3 and 4. This is done in Sections 3 and 4, respectively.

Moving forward, we will not distinguish between an operator $A$ acting on a finite-dimensional space and its matrix representation with respect to an orthonormal basis. The invariance of $W(A)$ under unitary similarities is the key to choosing the simplest matrix form possible.

The other ingredient of the reasoning involves the notion of the so called numerical range generating (or Kippenhahn) polynomial $P_{A}(\theta, \lambda)$ of $A$. This, by definition, is the characteristic polynomial of $\operatorname{Re}\left(e^{i \theta} A\right)$, where, as usual, $\operatorname{Re} X$ stands for the hermitian part of $X$. Denoting by $\lambda_{j}(\theta)$ the roots of this polynomial, recall that

$$
\left\{e^{-i \theta}\left(\lambda_{j}(\theta)+i \mathbb{R}\right): j=1, \ldots, n ; \theta \in(-\pi, \pi]\right\},
$$

is the family of tangent lines to a certain algebraic curve $C(A)$, the convex hull of which is $W(A)$, as was for the first time observed in [4] (see also its more accessible translation [5]). Respectively, $C(A)$ is called the numerical range generating curve, or the Kippenhahn curve, of $A$.

We state the pertinent (well known and also easily verifiable) result for convenience of reference.

Lemma 2.2. The Kippenhahn curve $C(X)$ of a given square matrix $X$ contains the circle of radius $r$ centered at the origin if and only if $P_{X}(\theta, \lambda)$ is divisible by $\lambda^{2}-r^{2}$ for all $\theta$.

In Section 3, assuming that $n=5$ and $\operatorname{rank} A=3$, we will show that there are no such $a \neq 0$ that make $P_{A-a I}$ divisible by $\lambda^{2}-r^{2}$ for some $r>0$, thus proving (GWW) in that case. For $\operatorname{rank} A=4$, the situation is slightly different, since then such $a$ and $r$ do exist. Instead, a general description of such matrices is given, and then it is observed that their numerical ranges are not circular nevertheless. This is happening since, for such matrices, $C(A)$ does not lie completely inside the circle $C=\{z:|z-a|=r\}$.

\section{Rank three partial isometries.}

Proposition 3.1. Let $A$ be a rank 3 partial isometry acting on a 5-dimensional space. Then $C(A)$ cannot contain a circle with a non-zero center.

Proof. We can choose an orthonormal basis in such a way that the respective matrix of $A$ (also denoted by $A$ ) has zeros in the first two columns, and the last three columns forming an orthonormal set. Invoke the Schur lemma to put the lower right 3-by-3 block of $A$ in an upper triangular form. Then use a unitary similarity of the upper left 2-by-2 block to set the $(2,3)$ entry of $A$ at zero.

Suppose now that $C(A) \supset\{z:|z-a|=r\}$ for some $a \in \mathbb{C}, r>0$. According to [8], $a$ is an eigenvalue of $A$ of algebraic multiplicity exceeding its geometric multiplicity. If $a \neq 0$, multiplying $A$ by a unimodular 
Electronic Journal of Linear Algebra, ISSN 1081-3810

A publication of the International Linear Algebra Society

Volume 38, pp. 107-113, January 2022

scalar (under which it remains a partial isometry), we may assume without loss of generality that $0<a \leq 1$. Furthermore, yet another (this time, diagonal) unitary similarity can be used to make the entries $a_{13}, a_{14}$, $a_{15}$ and $a_{24}$ of $A$ non-negative. As a result,

$$
A=\left[\begin{array}{ccccc}
0 & 0 & \sqrt{1-a^{2}} & t a & s a \\
0 & 0 & 0 & u & v \\
0 & 0 & a & -t \sqrt{1-a^{2}} & -s \sqrt{1-a^{2}} \\
0 & 0 & 0 & a & w \\
0 & 0 & 0 & 0 & z
\end{array}\right]
$$

where $s, t, u=\sqrt{1-a^{2}-t^{2}} \geq 0, s^{2}+|v|^{2}+|w|^{2}+|z|^{2}=1$ and

$$
s t+u v+a w=0 .
$$

Let now

$$
X:=A-a I=\left[\begin{array}{ccccc}
-a & 0 & \sqrt{1-a^{2}} & t a & s a \\
0 & -a & 0 & u & v \\
0 & 0 & 0 & -t \sqrt{1-a^{2}} & -s \sqrt{1-a^{2}} \\
0 & 0 & 0 & 0 & w \\
0 & 0 & 0 & 0 & z-a
\end{array}\right]
$$

and write the Kippenhahn polynomial of $X$ as

$$
P_{X}(\theta, \lambda)=-\lambda^{5}+\sum_{j=0}^{4} A_{j}(\theta) \lambda^{j} .
$$

The explicit formulas for $A_{j}$, obtained with the use of SageMath, are rather cumbersome and thus relegated to the Appendix.

Since $C(X)=C(A)-a$ contains a circle centered at the origin, according to Lemma 2.2, the radius $r$ of this disk is such that $P_{X}(\theta, \pm r)=0$. Equivalently,

$$
A_{0}+A_{2} r^{2}+A_{4} r^{4}=0 \text { and } A_{1}+A_{3} r^{2}-r^{4}=0 .
$$

With the use of the formulas for $A_{j}$, the equations (3.3) take the form

$$
b_{1} \cos \theta+b_{2} \sin \theta+b_{3} \cos ^{3} \theta+b_{4} \cos ^{2} \theta \sin \theta+b_{5} \cos ^{2} \theta=0,
$$

and

$$
c_{0}+c_{1} \cos \theta \sin \theta+c_{2} \cos ^{2} \theta=0
$$

where the coefficients $b_{j}, c_{j}$ depend on the matrix $A$ and on $r$, but not on $\theta$. We will provide the explicit expression only for those of them used in the reasoning below.

Since the function system

$$
\left\{1, \cos \theta, \sin \theta, \cos ^{2} \theta, \cos ^{2} \theta \sin \theta, \cos ^{3} \theta\right\}
$$

is linearly independent, equations $(3.4)$, (3.5) can only hold for all values of $\theta$ if the coefficients $b_{j}, c_{j}$ are equal to zero. But

$$
b_{3}=r^{2} a^{2}(\operatorname{Re} z-a), \quad b_{4}=-r^{2} a^{2} \operatorname{Im} z
$$


so that $z=a$. Substituting this into the formulas for $A_{j}$ and evaluating $c_{2}$, we get

$$
c_{2}=-a^{2} r^{2}+\frac{a}{4} \operatorname{Im}(w)(u \operatorname{Im}(v)+a \operatorname{Im}(w))+\frac{a}{4} \operatorname{Re}(w)(s t+u \operatorname{Re}(v)+a \operatorname{Re}(w)) .
$$

Rewriting (3.1) as

$$
u \operatorname{Im} v+a \operatorname{Im} w=0, \quad s t+u \operatorname{Re} v+a \operatorname{Re} w=0,
$$

we see that all the summands in the right hand side of (3.6), except for the very first one, cancel out. Hence $c_{2}=-a^{2} r^{2}=0$, a contradiction to $a, r>0$.

4. Rank four partial isometries. As it happens, a rank four 5-by-5 partial isometry $A$ can have a circle with a non-zero center as part of its Kippenhahn curve. The location of this center defines $A$ up to a unitary similarity. The radius of this circle is therefore defined uniquely. More specifically, the following statement holds.

Proposition 4.1. Let $A$ be a rank four 5-by-5 partial isometry. Then $C(A)$ contains a circle centered at ae $e^{i \omega}$ for some $a>0$ if and only if $a<1$ and $A$ is unitarily similar to

$$
e^{i \omega}\left[\begin{array}{ccccc}
0 & \sqrt{1-a^{2}} & a \sqrt{1-a^{2}} & a^{2} \sqrt{1-a^{2}} & a^{3} \sqrt{1-a^{2}} \\
0 & a & a^{2}-1 & a\left(a^{2}-1\right) & a^{2}\left(a^{2}-1\right) \\
0 & 0 & a & a^{2}-1 & a\left(a^{2}-1\right) \\
0 & 0 & 0 & a & a^{2}-1 \\
0 & 0 & 0 & 0 & a
\end{array}\right] .
$$

If this is the case, then the radius of the circle in question is $r=\left(1-a^{2}\right) / 2$.

Proof. Necessity. Choose an upper triangular matrix representation of $A$, with the zero first column and the remaining four forming an orthonormal set. Without loss of generality, we may (and, to simplify the notation, will) suppose that the center of the circle contained in $C(A)$ is positive, i.e. $e^{i \omega}=1$. At least two of the eigenvalues of $A$ are equal to $a$. Applying a permutational similarity if needed, we may set $a_{22}=a_{33}=a$. Also, as in the proof of Proposition 3.1, the first row of $A$ may be arranged to become entrywise non-negative. The orthonormality of the second and the third columns of $A$ and their orthogonality to the fourth and the fifth then yield

$$
A=\left[\begin{array}{ccccc}
0 & \sqrt{1-a^{2}} & a \sqrt{1-a^{2}} & x a \sqrt{1-a^{2}} & y a \sqrt{1-a^{2}} \\
0 & a & a^{2}-1 & x\left(a^{2}-1\right) & y\left(a^{2}-1\right) \\
0 & 0 & a & x\left(a^{2}-1\right) / a & y\left(a^{2}-1\right) / a \\
0 & 0 & 0 & u & v \\
0 & 0 & 0 & 0 & w
\end{array}\right]
$$

with $x, y \geq 0$ and some, yet to be determined, parameters $u, v, w \in \mathbb{C}$.

It is time now to invoke Lemma 2.2, according to which the Kippenhahn polynomial $P_{X}$ of $X=A-a I$ is divisible by $\lambda^{2}-r^{2}$ for some $r>0$. Writing $P_{X}$ as

$$
P_{X}(\theta, \lambda)=-\lambda^{5}+\sum_{j=0}^{4} B_{j}(\theta) \lambda^{j},
$$

we conclude that

$$
B_{0}+B_{2} r^{2}+B_{4} r^{4}=0 \text { and } \quad B_{1}+B_{3} r^{2}-r^{4}=0
$$


for all values of $\theta$. Using the explicit formulas for $B_{j}$ (obtained, as in Section 3, with the use of SageMath, and listed in the Appendix) observe that

$$
\begin{gathered}
B_{0}+B_{2} r^{2}+B_{4} r^{4}=b_{1} e^{i \theta}+b_{2} e^{-i \theta}+b_{3} e^{3 i \theta}+b_{4} e^{-3 i \theta}, \\
B_{1}+B_{3} r^{2}-r^{4}=c_{0}+c_{1} e^{2 i \theta}+c_{2} e^{-2 i \theta}
\end{gathered}
$$

with $b_{j}, c_{j}$ expressed in terms of $a, x, y, u, v, w$ and $r$. It follows from (4.9) that all $b_{j}, c_{j}$ are equal to zero. Now we have

$$
b_{3}=\frac{1}{8}\left(a^{2} r^{2} u+a^{2} r^{2} w-a^{3} r^{2}-a r^{2} u w\right)
$$

and

$$
c_{1}=\frac{1}{8}\left(-a^{4}-6 a^{2} r^{2}-\left(a^{2}+2 r^{2}-1\right) u w+a^{2}+\left(a^{3}+4 a r^{2}-a\right)(u+w)\right)
$$

Since $b_{3}$ can be rewritten as $-\frac{1}{8} a r^{2}(u-a)(w-a)$, either $u=a$ or $w=a$. Assuming the latter, (4.10) simplifies to $c_{1}=\frac{1}{4} a r^{2}(u-a)$, and so $u=a$ as well. Similarly, if $u=a$, then $c_{1}=0$ implies $w=a$. So, in fact $u=w=a$. Plugging these values of $u, w$ into (4.8) we obtain

$$
A=\left[\begin{array}{ccccc}
0 & \sqrt{1-a^{2}} & a \sqrt{1-a^{2}} & x a \sqrt{1-a^{2}} & y a \sqrt{1-a^{2}} \\
0 & a & a^{2}-1 & x\left(a^{2}-1\right) & y\left(a^{2}-1\right) \\
0 & 0 & a & x\left(a^{2}-1\right) / a & y\left(a^{2}-1\right) / a \\
0 & 0 & 0 & a & v \\
0 & 0 & 0 & 0 & a
\end{array}\right] .
$$

The unit length of column four implies now that $x=a$. Given this, the orthogonality of the last two columns allows us to express $v$ in terms of $y$ as $v=y\left(a^{2}-1\right) / a^{2}$. But the fifth column also has to have length one, so $y=a^{2}$ and $v$ simplifies to $a^{2}-1$. We have thus arrived at (4.7) with $\omega=0$.

To compute $r$, let us use the expression for $b_{1}\left(=b_{2}\right)$ which for the matrix under consideration takes the form

$$
b_{1}=\frac{1}{32}\left(a^{9}-4 a^{7}+6 a^{5}-16 a r^{4}-4 a^{3}+a\right),
$$

and, up to an inconsequential constant multiple, factors as

$$
\left(a^{4}-2 a^{2}+4 r^{2}+1\right)\left(a^{2}+2 r-1\right)\left(a^{2}-2 r-1\right) a .
$$

Since $a \leq 1$ and $r>0$, this product vanishes if and only if $r=\left(1-a^{2}\right) / 2$.

Sufficiency. Direct computations show that, for $A$ given by (4.7) with $\omega=0,0<a<1$ and $r=\left(1-a^{2}\right) / 2$, the Kippenhahn polynomial of $X=A-a I$ factors as

$$
-\left(\lambda^{2}-r^{2}\right)\left(\lambda^{3}+\sqrt{1-2 r}(\cos \theta) \lambda^{2}+\left(r^{2}-2 r\right) \lambda+r^{2} \sqrt{1-2 r} \cos \theta\right) .
$$

Note that the eigenvalue $e^{i \omega} a$ of the matrix (4.7) has geometric multiplicity one while the zero eigenvalue is not reducing. Consequently, this matrix is unitarily irreducible while its Kippenhahn polynomial is factorizable.

An explicit description of $W(A)$ for matrices (4.7) can be obtained with the use of (4.11). For our purposes, however, it suffices to observe that it differs from $D:=\left\{z:|z-a| \leq\left(1-a^{2}\right) / 2\right\}$, and thus is not a 
circular disk. To this end, recall that the numerical range of any matrix contains the numerical ranges of its principal submatrices. In particular, $W(A)$ contains the numerical range of its upper left 2-by-2 block. By the Elliptical Range Theorem (and, once again, setting for simplicity $\omega=0$ ), the latter is the elliptical disk with the foci 0 and $a$ and the minor axis of length $\sqrt{1-a^{2}}$. The leftmost point of this ellipse is $(a-1) / 2$, and its distance from $a$ is $(1+a) / 2>r$.

This observation, combined with Propositions 2.1, 3.1, and 4.1, completes the proof of Theorem 1.1.

More can be said about the shape of $W(A)$ of matrices (4.7). In particular, denoting the second factor of (4.11) by $p(\lambda)$, observe that

$$
p( \pm r)=2 r^{2}(\sqrt{1-2 r} \cos \theta \pm(r-1))
$$

Consequently, $p(r)<0, p(-r)>0$, and so $p$ has roots both smaller than $-r$ and larger than $r$. In other words, $D$ lies in the interior of $W(A)$.

Appendix. The coefficients of $P_{X}$ in the rank three case are

$$
\begin{aligned}
& A_{0}=a_{01} \cos \theta+a_{02} \sin \theta, \quad \text { where } \\
& a_{01}:=\frac{1}{16}\left(a^{2}-1\right)\left(a u^{2}+a|w|^{2}+u \operatorname{Re}(v \bar{w})-u^{2} \operatorname{Re}(z)\right) \\
& a_{02}:=\frac{1}{16}\left(a^{2}-1\right)\left(u \operatorname{Im}(v \bar{w})+u^{2} \operatorname{Im}(z)\right), \\
& A_{1}=a_{10}+a_{11} \cos \theta \sin \theta+a_{12} \cos ^{2} \theta, \quad \text { where } \\
& a_{10}:=\frac{1}{16}\left(\left(a^{2}-1\right)\left(u^{2}+|v|^{2}+|w|^{2}\right)+2 s t u \operatorname{Re}(v)-t^{2}|v|^{2}-s^{2} u^{2}\right) \\
& a_{11}:=\frac{1}{4} a\left(\left(a^{2}-t^{2}-u^{2}-1\right) \operatorname{Im}(z)+s t \operatorname{Im}(w)+u \operatorname{Im}(w \bar{v})\right) \\
& a_{12}:=\frac{1}{4} a\left(\left(a^{2}-t^{2}-u^{2}-1\right)(\operatorname{Re}(z)-a)+u \operatorname{Re}(v \bar{w})+a|w|^{2}+s t \operatorname{Re}(w)\right), \\
& A_{2}=a_{21} \cos \theta+a_{22} \sin \theta+a_{23} \cos ^{2} \theta \sin \theta+a_{24} \cos ^{3} \theta, \quad \text { where } \\
& a_{21}:=\frac{1}{4}\left(a\left(s^{2}+|v|^{2}+2|w|^{2}\right)+\left(a^{2}-t^{2}-u^{2}-1\right)(\operatorname{Re} z-2 a)+s t \operatorname{Re} w+u \operatorname{Re}(v \bar{w})\right) \\
& a_{22}:=\frac{1}{4}\left(\left(t^{2}+u^{2}+1-a^{2}\right) \operatorname{Im}(z)-s t \operatorname{Im}(w)+u \operatorname{Im}(v \bar{w})\right) \\
& a_{23}::=-a^{2} \operatorname{Im}(z) \\
& a_{24}:=a^{2}(\operatorname{Re}(z)-a), \\
& A_{3}=a_{30}+a_{31} \cos { }^{2} \theta+a_{32} \cos \theta \sin \theta, \quad \text { where } \\
& a_{30}:=\frac{1}{4}\left(s^{2}+t^{2}+u^{2}+|v|^{2}+|w|^{2}+1-a^{2}\right) \\
& a_{31}:=a(2 \operatorname{Re}(z)-3 a) \\
& a_{32}:=-2 a \operatorname{Im}(z), \\
& A_{4}=(\operatorname{Re}(z)-3 a) \cos \theta-\operatorname{Im}(z) \sin \theta .
\end{aligned}
$$


The coefficients of $P_{X}$ in the rank four case are

$$
\begin{aligned}
B_{0}= & b_{01} e^{i \theta}+\overline{b_{01}} e^{-i \theta}, \quad \text { where } \\
b_{01}:= & \frac{1}{32} \frac{\left(1-a^{2}\right)^{3}}{a^{2}}\left(w x^{2}+u y^{2}-a x^{2}-a y^{2}-v x y\right) \\
B_{1}= & b_{10}+b_{12} e^{2 i \theta}+\overline{b_{12}} e^{-2 i \theta}, \quad \text { where } \\
b_{10}:= & \frac{1}{4}\left(1-a^{2}\right)\left(a^{2}-a \operatorname{Re} u-a \operatorname{Re} w+\operatorname{Re}(w \bar{u})-\frac{|v|^{2}}{2}\right)-\frac{\left(1-a^{2}\right)^{3}}{16 a^{2}}\left(x^{2}+y^{2}\right) \\
& b_{12}:=\frac{1}{8}\left(1-a^{2}\right)\left(a^{2}-a u-a w+u w\right) \\
B_{2}= & b_{21} e^{i \theta}+\overline{b_{21}} e^{-i \theta}+b_{23} e^{3 i \theta}+\overline{b_{23}} e^{-3 i \theta}, \quad \text { where } \\
& b_{21}:=\frac{1-a^{2}}{8 a^{2}}\left(v x y-w x^{2}-u y^{2}\right)+\frac{1-a^{2}}{8 a}\left(4 a^{2}-2 a u-2 a w+x^{2}+y^{2}\right) \\
& +\frac{a^{2}}{8}(u+w+2 \operatorname{Re} u+2 \operatorname{Re} w-3 a)+\frac{a}{8}\left(|v|^{2}-2 w \operatorname{Re} u-u \bar{w}\right) \\
B_{43}:= & \frac{1}{8}\left(a^{2} u+a^{2} w-a u w-a^{3}\right) \\
B_{3}= & b_{30}+b_{32} e^{2 i \theta}+\overline{b_{32}} e^{-2 i \theta}, \quad \text { where } \\
& b_{30}:=\frac{1}{4}\left(2-8 a^{2}-x^{2}-y^{2}+4 a \operatorname{Re}(u+w)-2 \operatorname{Re}(u \bar{w})+|v|^{2}+\frac{x^{2}}{a^{2}}+\frac{y^{2}}{a^{2}}\right) \\
& b_{32}:=\frac{1}{4}\left(2 a u+2 a w-u w-3 a^{2}\right) \\
1 & =\frac{1}{2}(\bar{u}+\bar{w}-3 a) e^{-i \theta} .
\end{aligned}
$$

Acknowledgment. The authors are thankful to the anonymous referee for carefully checking the formulas and pointing out a flaw in the coefficient $b_{1}$ as calculated from the original version of the appendix.

\section{REFERENCES}

[1] H.-L. Gau, K.-Z. Wang, and P.Y. Wu. Circular numerical ranges of partial isometries. Linear Multilinear Algebra, 64(1):14$35,2016$.

[2] K.E. Gustafson and D.K.M. Rao. Numerical Range. The Field of Values of Linear Operators and Matrices. Springer, New York, 1997.

[3] R.A. Horn and C. R. Johnson. Topics in Matrix Analysis. Cambridge University Press, Cambridge, 1994, Corrected reprint of the 1991 original.

[4] R. Kippenhahn. Über den Wertevorrat einer Matrix. Math. Nachr., 6:193-228, 1951.

[5] R. Kippenhahn. On the numerical range of a matrix. Linear Multilinear Algebra, 56(1-2):185-225, 2008. Translated from the German by Paul F. Zachlin and Michiel E. Hochstenbach.

[6] M. Naimi and M. Benharrat. On the circular numerical ranges of 5-by-5 partial isometries. arXiv.math.FA/2108.04459v1, $1-17,2021$.

[7] E. Wegert and I.M. Spitkovsky. On partial isometries with circular numerical range. Concr. Oper., 8:176-186, 2021.

[8] P.Y. Wu. Numerical ranges as circular discs. Appl. Math. Lett., 24(12):2115-2117, 2011. 